\title{
Vlasov versus reduced kinetic theories for helically symmetric equilibria
}

\author{
H. Tasso ${ }^{1}$, G. N. Throumoulopoulos ${ }^{2}$ \\ ${ }^{1}$ Max-Planck-Institut für Plasmaphysik, Euratom Association, \\ D-85748 Garching, Germany \\ ${ }^{2}$ University of Ioannina, Association Euratom-Hellenic Republic, \\ Section of Theoretical Physics, GR 45110 Ioannina, Greece
}

\begin{abstract}
A new constant of motion for helically symmetric equilibria in the vicinity of the magnetic axis is obtained in the framework of Vlasov theory. In view of this constant of motion the Vlasov theory is compared with drift kinetic and gyrokinetic theories near axis. It turns out that as in the case of axisymmetric equilibria [H. Tasso and G. N. Throumoulopoulos, Phys. Plasmas 18, 064507 (2011)] the Vlasov current density thereon can differ appreciably from the drift kinetic and gyrokinetic current densities. This indicates some limitation on the implications of reduced kinetic theories, in particular as concerns the physics of energetic particles in the central region of magnetically confined plasmas.
\end{abstract}

Accepted for publication in Physics of Plasmas 


\section{Introduction}

Kinetic equilibria may provide broader and more precise information than multifluid or magnetogydrodymanic equilibria as those governed by the GradShafranov equation. Since solving self consistently the kinetic equations is tough particularly in complicated geometries the majority of kinetic equilibrium solutions are restricted to one dimensional configurations in plane geometry, e.g. [1]-8]. Of particular interest are equilibria with sheared toroidal and poloidal flows which play a role in the transition to improved confinement regimes in tokamaks and stellarators, though understanding the physics of this transition remains incomplete. Construction of kinetic equilibria is crucially related to the particle constants of motion which the distribution function depends on. In the framework of Maxwell-Vlasov theory only a couple of constants of motions are known for symmetric two-dimensional equilibria, i.e. the energy, $H$, and the momentum $p_{x 3}$ conjugate to the ignorable coordinate, $x_{3}$, out of the four potential constants of motion. Therefore, for distribution functions of the form $f\left(H, p_{x 3}\right)$ only macroscopic flows and currents along the direction associated with $x_{3}$ can be derived, e.g. toroidal flows for axisymmetric plasmas. The creation of poloidal flows requires additional constant(s) of motion. This remains an open question despite the fact that in a previous study [9] we found locally in the vicinity of the magnetic axis the following new constant of motion: $C=v_{z}+I \ln \left|v_{\phi}\right|$, where $v_{\phi}$ is the toroidal particle velocity, $v_{z}$ the velocity component parallel to the axis of symmetry and $\mathbf{B}_{\phi}=I / r \mathbf{e}_{\phi}$ the toroidal magnetic field near axis $(r, \phi, z$ are cylindrical coordinates). An additional open question remains a potential extension of the proof of non existence of magnetohydrodynamic axisymmetric equilibria with purely poloidal flows [10] to Vlasov equilibria. A third constant of motion was studied extensively in the Astrophysics literature [11]-[24]. In particular for axisymmetric astrophysical systems distribution functions depending on an approximate third constant of motion result in velocity ellipsoids with unequal axes in agreement with observational data for our Galaxy [14, 17, 24].

Owing to the contemporary and probably future limited computational efficiency for simulations in the framework of Vlasov theory approximate kinetic theories have been established in reduced phase spaces as the drift kinetic and gyrokinetic ones. In both theories the reduced phase space is five dimensional with three spatial components associated with the guiding center position, $\mathbf{R}$ (or the gyrocenter position in the framework of gyrokinetic 
theory), and a velocity component parallel to the magnetic field, $v_{\|}$; also, the two components of the perpendicular particle velocity are approximated after a gyroangle averaging with the magnetic moment which is treated as an adiabatic invariant. A related underlying assumption for both reduced theories is that the ratio $\epsilon$ of the gyroperiod to the macroscopic time scale is small. In the drift kinetic theory $\epsilon$ is the same as the ratio of the gyroradius to macroscopic scale length while in the gyrokinetic theory small spatial variations are permitted. Because of the reduction of the phase space some information of the particle motion is missed. In this respect it may be noted that the reduced-phase-space kinetic theories are developed via expansions in $\epsilon$, the convergence of which is not guaranteed. This gives rise to the question: is the missing information important? To address this question we compared axisymmetric Vlasov equilibria near the magnetic axis with drift kinetic and gyrokinetic ones [25]. It turned out that because of missing the above constant of motion, $\mathrm{C}$, in the latter case the on axis current density can differ appreciably from the "actual" Vlasov current density. Also, unlikely Vlasov theory, the reduced kinetic theories can not distinguish a straight from a curved magnetic axis.

The aim of the present contribution is twofold: first to extend the above local constant of motion, $C$, to the more general class of helical symmetric equilibria and second to compare the Vlasov helical symmetric equilibrium characteristics near the magnetic axis with respective drift kinetic and gyrokinetic characteristics. The new local constant of motion is derived in Sec. II. Then, Vlasov theory is compared near axis with reduced kinetic theories in Sec. III. Sec. IV summarizes the conclusions.

\section{A third Vlasov constant of motion near magnetic axis}

We will employ the following form of Vlasov equation [26]:

$$
\frac{\partial f}{\partial t}+\mathbf{v} \cdot \nabla f+\mathbf{e}_{i} \cdot(\mathbf{E}+\mathbf{v} \times \mathbf{B}) \frac{\partial f}{\partial v_{i}}+\left(\mathbf{e}_{i} \cdot \mathbf{v} \times \nabla \times \mathbf{v}\right) \frac{\partial f}{\partial v_{i}}=0 .
$$

To derive (11) one uses general orthogonal coordinates $\left(x_{1}, x_{2}, x_{3}\right)$ with unit basis vectors $\mathbf{e}_{i}=\boldsymbol{\nabla} x_{i} /\left|\boldsymbol{\nabla} x_{i}\right|(i=1,2,3)$, expresses the "microscopic fluid" velocity in the basis of $\mathbf{e}_{i}$ as $\mathbf{v}=v_{i} \mathbf{e}_{i}$ and uses the "microscopic fluid" momentum equation,

$$
\frac{\partial \mathbf{v}}{\partial t}+\mathbf{v} \cdot \nabla \mathbf{v}=\mathbf{E}+\mathbf{v} \times \mathbf{B}
$$


where $\mathbf{E}$ and $\mathbf{B}$ are the electric and magnetic fields consistent with Maxwell equations. The term "microscopic fluid" relates to the fact that the Vlasov equation is an approximation to the N-particles Liouville equation, which replaces the $\mathrm{N}$ particles by a continuum. This is sometimes termed "fluid approximation". To avoid confusion, however, the usual term "particle" will be adopted in place of "microscopic fluid". Also, for the sake of notation simplicity and without loss of generality we will consider only ions and employ convenient units by setting $m=q=c=1$ where $m$ and $q$ are the ion mass and charge and $c$ is the velocity of light.

In connection with the helical symmetry we specify the coordinates as $\left(x_{1}=r, x_{2}=a \phi+\beta z, x_{3}=-\beta \phi+a z\right)$, where $a$ and $\beta$ are parameters. Helical symmetry means that any quantity does not depend on $x_{3}$. Translational symmetry and axisymmetry are then recovered as particular cases for $(a=1$, $\beta=0)$ and $(a=0, \beta=1)$. The respective unit vectors are

$$
\mathbf{e}_{1}=\mathbf{e}_{r}, \quad \mathbf{e}_{2}=\frac{a \mathbf{e}_{\phi}+\beta r \mathbf{e}_{z}}{\left(a^{2}+\beta^{2} r^{2}\right)^{1 / 2}}, \quad \mathbf{e}_{3}=\frac{a r \mathbf{e}_{z}-\beta \mathbf{e}_{\phi}}{\left(\beta^{2}+a^{2} r^{2}\right)^{1 / 2}} .
$$

Note that these basis vectors are in general non orthogonal. A helical magnetic axis however is located at a constant distance $r$ from the $z$-axis. Since we will study the equilibrium near the magnetic axis we set $r=1$ thereon and make the choice $a^{2}+\beta^{2}=1$. Consequently, the above basis vectors reduce to $\left(\mathbf{e}_{1}=\mathbf{e}_{r}, \mathbf{e}_{2}=a \mathbf{e}_{\phi}+\beta \mathbf{e}_{z}, \mathbf{e}_{3}=-\beta \mathbf{e}_{\phi}+a \mathbf{e}_{z}\right)$ and become orthogonal on axis, with $\mathbf{e}_{2}$ along and $\mathbf{e}_{3}$ perpendicular to the axis. Thus, (11) can be applied in the neighbourhood of axis. The magnetic field near axis can be written in the form

$$
\mathbf{B}=\frac{I}{r} \mathbf{e}_{\phi}+B_{0} \mathbf{e}_{z}=\frac{I}{r} \frac{a \mathbf{e}_{2}-\beta \mathbf{e}_{3}}{a^{2}+\beta^{2}}+B_{0} \frac{\beta \mathbf{e}_{2}+a \mathbf{e}_{3}}{a^{2}+\beta^{2}} .
$$

By the choice $a B_{0}=\beta I$ we take on axis $\mathbf{B}=I / a \mathbf{e}_{2}$ as it should be. Also, we examine the case of $\mathbf{E}=0$ on axis but $\nabla f \neq 0$ thereon. The latter assumption may be regarded as extraordinary because $\nabla f$ is related to the density gradient on axis which for usual peaked density profiles is expected to vanish. The reason for assuming $\nabla f \neq 0$ on axis is that for axisymmetric equilibria, if (11) is solved near axis by the method of characteristics under the assumption $\nabla f=0$ on axis the usual toroidal momentum constant of motion, $p_{\phi}$, is missed, while $p_{\phi}$ is recovered if $\nabla f \neq 0$ thereon [25]. 
Using the above basis for the helically symmetric equilibria under consideration Eq. (1) near axis becomes

$$
v_{1} \frac{\partial f}{\partial x_{1}}+v_{2} \frac{\partial f}{\partial x_{2}}+w_{1} \frac{\partial f}{\partial v_{1}}+w_{2} \frac{\partial f}{\partial v_{2}}+w_{3} \frac{\partial f}{\partial v_{3}}=0
$$

where

$w_{1}=\left(a v_{2}-\beta v_{3}\right)^{2}-v_{3} \frac{I}{a}, w_{2}=a v_{1}\left(\beta v_{3}-a v_{2}\right), w_{3}=v_{1} \frac{I}{a}+v_{1} \beta\left(a v_{2}-\beta v_{3}\right)$.

The characteristics of (2) are given by the solutions of

$$
\frac{d x_{1}}{d v_{1}}=\frac{d x_{2}}{d v_{2}}=\frac{d v_{1}}{d w_{1}}=\frac{d v_{2}}{d w_{2}}=\frac{d v_{3}}{d w_{3}} .
$$

Integration of the last equation yields the new constant of motion:

$$
\begin{aligned}
C= & v_{3}+\frac{\beta\left(\beta^{2}-a^{2}\right)\left(\beta v_{3}-a v_{2}\right)+a I \ln \left|\left[a^{4} v_{2}-a^{2} \beta^{2} v_{2}+a \beta^{3} v_{3}-\beta\left(I+a^{3} v_{3}\right)\right]\right|}{\left(a^{2}-\beta^{2}\right)^{2}} \\
& \quad \text { for } a \neq \beta, \\
C= & 2 v_{3}-v_{2}-\frac{a^{3}}{I} \frac{\left(v_{2}-v_{3}\right)^{2}}{2} \text { for } a=\beta .
\end{aligned}
$$

The respective axisymmetric constant of motion, $C=v_{z}+I \ln \left|v_{\phi}\right|$, is recovered from (5) for $(a=1, \beta=0)$ and the translational symmetric constant of motion, $C=v_{z}$, for $(a=0, \beta=1)$. Also, conservation of energy $H=1 / 2\left(v_{1}^{2}+v_{2}^{2}+v_{3}^{2}\right)$ follows from the manipulation of equations (44) presented in Appendix. Distribution functions of the form $f(H, C)$ could create a helical current on axis because of the dependence of $C$ on $v_{2}$ additionally to that in the logarithmic term. This is different from the axisymmetric case in which toroidal axisymmetric currents on axis are not possible at all for the same class of distribution functions because of the dependence of $C$ on $\left|v_{\phi}\right|$. This difference may be expected because for translational symmetric equilibria which are recoverable from the helically symmetric ones the respective distribution functions $f(H, C)=f\left(H, v_{z}\right)$ can create currents on axis. However, such a helical equilibrium is not physically acceptable because of the dependence of $C$ on $v_{3}$ (but not on $v_{1}$ ) implying the creation of a flow perpendicular to the axis. Similar unphysical flows are created in the axisymmetric case because of the respective dependence of $C$ on $v_{z}$ (but not on 
$v_{r}=v_{1}$ ). As discussed in 9 possible reasons of this unphysical behaviour are: (i) the lack of a potential fourth constant of motion involving $v_{1}$ in such a way that creation of regular poloidal flows be possible, (ii) non taking into account here the MHD property of coincidence of the magnetic surfaces with the flow surfaces, (iii) additional drifts because of the curvature and torsion of the magnetic field which are eliminated in translational symmetric geometry and (iv) potential damping of unphysical flows in the framework of a collisional kinetic theory. The above consideration shows that straight, circular and helical magnetic axes are well distinguished in the framework of Vlasov theory.

Unlikely the axisymmetric case [25] the generalized momentum constant of motion, $p_{x 3}=v_{3}+A_{3}$, where $\mathbf{A}\left(x_{1}, x_{2}\right)$ is the vector potential, can not follow from (4) though we have assumed $\boldsymbol{\nabla} f \neq 0$ on axis. This is obtained form the Lagrangian

$$
L=\frac{1}{2}\left(\dot{x}_{1}^{2}++\dot{x}_{2}^{2}+\dot{x}_{3}^{2}\right)+\Phi\left(x_{1}, x_{2}\right)+\dot{\mathbf{x}} \cdot \mathbf{A}\left(x_{1}, x_{2}\right)
$$

even in the presence of an electric filed on axis associated with the electrostatic potential $\Phi$. Also, a generic non local derivation off $p_{x 3}$ is provided in [27].

\section{Comparison of Vlasov with drift kinetic and gyrokinetic theories.}

The near axis consideration of helically symmetric equilibria will be repeated in the framework of the drift kinetic and gyrokinetic theories which were employed in Ref. [25] on an individual basis below. For convenience a brief review of these theories will also be given here because otherwise frequent reference to [25] would make reading of the present section tedious.

\section{Drift kinetic theory}

The drift kinetic theory established in Ref. [31] is based on the Littlejohn's Lagrangian for the guiding center motion [32] extended to include the polarization drift in such a way that local conservation of energy is guaranteed. Introducing the modified potentials, $\mathbf{A}^{\star}$ and $\Phi^{\star}$, this Lagrangian can be written in the concise form 33

$$
L=\mathbf{A}^{\star} \cdot \dot{\mathbf{R}}-\Phi^{\star},
$$


where

$$
\begin{aligned}
\mathbf{A}^{\star} & =\mathbf{A}+v_{\|} \mathbf{b}+\mathbf{V}_{E} \\
\Phi^{\star} & =\Phi+\mu B+\frac{1}{2}\left(v_{\|}^{2}+v_{E}^{2}\right) \\
\mathbf{V}_{E} & =\frac{\mathbf{E} \times \mathbf{B}}{B^{2}}
\end{aligned}
$$

Here, $\Phi$ and $\mathbf{A}$ are the usual electromagnetic scalar and vector potentials and $\mathbf{b}=\mathbf{B} / B$. The drift kinetic equation for the guiding center distribution function $f\left(\mathbf{R}, v_{\|}, \mu, t\right)$ (with $\dot{\mu}=0$ ) acquires the form

$$
\frac{\partial f}{\partial t}+\mathbf{V} \cdot \nabla f+\dot{v}_{\|} \frac{\partial f}{\partial v_{\|}}=0 .
$$

By means of the Euler-Lagrange equations following from (7) the guiding center velocity, $\mathbf{V}$, and the "acceleration" parallel to the magnetic field, $\dot{v}_{\|}$, can be expressed as:

$$
\begin{aligned}
& \mathbf{V}=\dot{\mathbf{R}}=v_{\|} \frac{\mathbf{B}^{\star}}{B_{\|}^{\star}}+\frac{\mathbf{E}^{\star} \times \mathbf{b}}{B_{\|}^{\star}}, \\
& \dot{v}_{\|}=\frac{\mathbf{E}^{\star} \cdot \mathbf{B}^{\star}}{B_{\|}^{\star}}=\frac{1}{v_{\|}} \mathbf{V} \cdot \mathbf{E}^{\star},
\end{aligned}
$$

where

$$
\begin{gathered}
\mathbf{E}^{\star}=-\frac{\partial \mathbf{A}^{\star}}{\partial t}-\frac{\partial \Phi^{\star}}{\partial \mathbf{R}}, \quad \mathbf{B}^{\star}=\boldsymbol{\nabla} \times \mathbf{A}^{\star}, \\
B_{\|}^{\star}=\mathbf{B}^{\star} \cdot \mathbf{b}=B+v_{\|} \mathbf{b} \cdot \boldsymbol{\nabla} \times \mathbf{b}+\mathbf{b} \cdot \boldsymbol{\nabla} \times \mathbf{V}_{E} .
\end{gathered}
$$

Explicit expressions for $\mathbf{V}$ and $\dot{v}_{\|}$are given by Eqs. (3.24) and (4.17) of Ref. [31. Also, it is noted here that Eqs. (12) and (13) have similar structure as the respective gyrokinetic equations of Refs. [38] and [39] [Eqs. (5.39) and (5.41) therein]. Since $B_{\|}^{\star}$ appears in the denominators of (12) and (13) a singularity occurs for $B_{\|}^{\star}=0$. For $\mathbf{E}=0$ this singularity can be expressed by the critical parallel velocity $v_{c}=-\Omega /(\mathbf{b} \cdot \nabla \times \mathbf{b})$, where $\Omega$ is the gyrofrequency. Therefore, the theory is singular for large $\left|v_{\|}\right|$at which $\mathbf{V}$ and $\dot{v}_{\|}$diverge and consequently non-casual guiding center orbits occur and the guiding center conservation in phase space is violated [30]. It is the $v_{\|}$-dependence of $\mathbf{A}^{\star}$ [Eq. (8) ] that produces the singularity. In order to regularize the singularity $v_{\|}$in (8) can be replaced by an antisymmetric function $g(z)$ with $z=v_{\|} / v_{0}$, where $v_{0}$ is some constant velocity [30, 31, 33]. The nonregularized theory 
presented here for simplicity is obtained for $g(z)=z$. In the regularized theory $g(z) \approx z$ should still hold for small $|z|$. For large $|z|$, however, $g$ must stay finite such that with $v_{0} \gg v_{\text {thermal }}$ one has $v_{0} g(\infty)<v_{c}$. A possible choice for $g(z)$ is $g(z)=\tanh z$.

Since for a helically symmetric equilibrium the magnetic field on axis becomes purely helical and dependent only on $r$, one readily calculates near axis with $\mathbf{E}=0$ thereon:

$$
\begin{gathered}
\nabla \times \mathbf{b}=\nabla \times \mathbf{e}_{2}=\frac{a}{r} \mathbf{e}_{z}, \quad \mathbf{B}^{\star}=\mathbf{B}+v_{\|} \frac{a}{r} \mathbf{e}_{z}, \quad B_{\|}^{\star}=B+v_{\|} \frac{a}{r} \beta \\
\nabla B(r)=\frac{d B}{d r} \mathbf{e}_{r}, \quad \Phi^{\star}=\mu B+\frac{1}{2} v_{\|}^{2}, \quad \mathbf{E}^{\star}=-\mu \frac{d B}{d r} \mathbf{e}_{r}
\end{gathered}
$$

and consequently

$$
\begin{gathered}
\mathbf{V}=v_{\|} \frac{B}{B^{\star} \|} \mathbf{e}_{2}+\frac{v_{\|}^{2} a}{r B_{\|}^{\star}} \mathbf{e}_{z}-\frac{\mu}{B_{\|}^{\star}} \frac{d B}{d r} \mathbf{e}_{3}, \\
\dot{v}_{\|}=0 .
\end{gathered}
$$

As expected on axis the guiding center velocity consists of a component parallel to B and the curvature and grad- $B$ drifts. Also, the "acceleration" $\dot{v}_{\|}$vanishes because there is no parallel force and the drift kinetic equation (11) becomes

$$
\mathbf{V} \cdot \frac{\partial f}{\partial \mathbf{R}}=0
$$

Because of (19) $v_{\|}$is a constant of motion and therefore distribution functions of the form $f\left(H, v_{\|}\right)$, where $H=\mu B+1 / 2 v_{\|}^{2}$, either can or can't produce helical currents by choosing $f$ either odd or even function of $v_{\|}$. This property holds also for axisymmetric and translational symmetric equilibria [25] because (19) keeps valid irrespective of the kind of symmetry. Thus, unlike Vlasov theory the drift kinetic theory can not distinguish equilibria of straight, circular or helical magnetic axes. Also, according to the results of section III the respective obtainable near axis Vlasov class of distribution functions $f(H, C)$ can not produce physically acceptable currents on axis 1 . Consequently, since near axis the overwhelming majority of the particles are

\footnotetext{
${ }^{1}$ Physically acceptable Vlasov currents can be produced by the class $f\left(H, p_{x 3}\right)$ which, however, corresponds to drift kinetic distribution functions of the form $f\left(H, p_{x 3}^{d}\right)$, where near axis $p_{x 3}^{d}=A_{3}+v_{\|} b_{3}$, as it follows from the Lagrangian (77).
} 
passing, the parallel currents constructed in the framework of the drift kinetic theory may differ from the "actual" ones. Also, unlike in the case of axisymmetric equilibria [25], the $B_{\|}^{\star}$-singularity is present as indicated by Eq. (16).

\section{Gyrokinetic theory}

We will use the gyrokinetic equations of Ref. 35] which have been employed to a variety of applications (see for example Refs. [40, 41, 42]). Eq. (11) remains identical in form where $f\left(\mathbf{R}, v_{\|}, \mu, t\right)$ is now the gyrocenter distribution function for ions. The gyrocenter velocity and "acceleration" are given by

$$
\begin{gathered}
\mathbf{V}=v_{\|} \mathbf{b}_{0}+\frac{B_{0}}{B_{0 \|}^{\star}}\left(\mathbf{V}_{E}+\mathbf{V}_{\boldsymbol{\nabla}_{B}}+\mathbf{V}_{c}\right), \\
\dot{v}_{\|}=-\frac{1}{v_{\|}} \mathbf{V} \cdot\left(\boldsymbol{\nabla} \bar{\Phi}+\mu \boldsymbol{\nabla} B_{0}\right) .
\end{gathered}
$$

Here, $\mathbf{B}_{0}$ is the equilibrium magnetic field, $\mathbf{b}_{0}=\mathbf{B}_{0} / B_{0}$,

$$
B_{0 \|}^{\star}=\left(\mathbf{B}_{0}+v_{\|} \nabla \times \mathbf{b}_{0}\right) \cdot \mathbf{b}_{0},
$$

$\bar{\Phi}$ stands for the perturbed gyroaveraged electrostatic potential, and the $\mathbf{E} \times \mathbf{B}$-drift velocity $\mathbf{V}_{E}$, the grad- $B$ drift velocity $\mathbf{V}_{\boldsymbol{\nabla}_{B}}$, and the curvature drift velocity $\mathbf{V}_{c}$ are given by

$$
\begin{gathered}
\mathbf{V}_{E}=-\frac{\nabla \bar{\Phi} \times \nabla B_{0}}{B_{0}^{2}}, \\
\mathbf{V}_{\nabla_{B}}=\frac{\mu}{\Omega B_{0}} \mathbf{B}_{0} \times \nabla B_{0}, \\
\mathbf{V}_{c}=\frac{\mu v_{\|}^{2}}{\Omega B_{0}^{2}} \mathbf{b}_{0} \times \nabla\left(p_{0}+\frac{B_{0}^{2}}{2}\right) .
\end{gathered}
$$

Note that as in the case of drift kinetic theory a similar singularity occurs at $B_{0 \|}^{\star}=0$. In numerical applications this singularity was "avoided" by approximating $B_{0 \|}^{\star}=B_{0}$ (see for example Refs. [40, 41]). Consideration of the above equations for a helically symmetric equilibrium with $\mathbf{E}=0$ near axis yields relations similar to (18), (19) and (20). Therefore, the above found discrepancies of the drift kinetic theory with the Vlasov one persist in the 
framework of the gyrokinetic theory. The structure of the reduced kinetic equations in conjunction with the symmetry of the equilibrium considered clearly indicate that this conclusion is independent of the particular drift kinetic or gyrokinetic equations.

\section{Summary}

We have found a new constant of motion near the magnetic axis of helically symmetric equilibria in the framework of Vlasov theory [Eqs. (5) and (6) ]. On account of this constant of motion a comparison of the Vlasov equation with either the drift kinetic or the gyrokinetic equation indicates that the current densities near the magnetic axis in the former case may be different from the ones in the latter case. Also, unlike Vlasov, the reduced kinetic theories can not distinguish equilibria with straight, circular or helical magnetic axes. This discrepancy is due to the loss of new Vlasov constant of motion in the reduced phase space thus indicating that this reduction, even if made rigorously so that local conservation laws and Liouvillean invariance of the volume element is guarantied, is associated with the loss of nontrivial physics. This could put certain limits on the conclusions from drift kinetic or gyrokinetic simulations for time scales large compared to the gyroperiod which is the case of an equilibrium. Though the derivations of both theories are formally correct the convergence of the $\epsilon$-expansions is presumably not uniform for all times which could lead to the noticed discrepancies calculated in this study.

In addition, a singularity which occurs in both drift kinetic and gyrokinetic theories for large parallel particle velocities, present for helically symmetric equilibria, is usually eliminated in the literature by a rough approximation. Alternative to the rather artificially imposed regularization reported in Sec. III, one might remove this singularity in the integrals associated with moments of the drift kinetic equations (see the generic relations (8.14)-(8.17) of [31] for the self consistent charge, current, energy and energy flux densities) by Cauchy principle value integration provided that such an integration would be physically justifiable. This requires further investigation.

\section{Appendix: Energy conservation near axis}

From

$$
\frac{d x_{1}}{v_{1}}=\frac{d v_{1}}{w_{1}} \text { and } \frac{d x_{2}}{v_{2}}=\frac{d v_{2}}{w_{2}}
$$


[see Eqs. (41)] it follows

$$
w_{1} d x_{1}+w_{2} d x_{2}=\frac{1}{2} d\left(v_{1}^{2}+v_{2}^{2}\right)
$$

Also, employing

$$
\frac{d x_{1}}{v_{1}}=\frac{d x_{2}}{v_{2}}=\frac{d v_{3}}{w_{3}}
$$

one has

$$
w_{1} d x_{1}+w_{2} d x_{2}=\left(w_{1} v_{1}+w_{2} v_{2}\right) \frac{d v_{3}}{w_{3}}
$$

Explicit calculation by means (31) yields

$$
w_{1} v_{1}+w_{2} v_{2}=-w_{3} v_{3}
$$

From (A1), (A2) and (A3) it follows

$$
\frac{1}{2}\left(v_{1}^{2}+v_{2}^{2}+v_{3}^{2}\right)=\text { const. }
$$

\section{Acknowledgements}

Part of this work was conducted during a visit of G.N.T. to the Max-PlanckInstitut für Plasmaphysik, Garching. The hospitality of that Institute is greatly appreciated.

This work was performed within the participation of the University of Ioannina in the Association Euratom-Hellenic Republic, which is supported in part by the European Union and by the General Secretariat of Research and Technology of Greece. The views and opinions expressed herein do not necessarily reflect those of the European Commission. 


\section{References}

[1] P. J. Channell, Phys. Fluids 19, 1541 (1976).

[2] S. M. Mahajan, Phys. Fluids B 1, 43 (1989).

[3] N. Attico, F. Pegoraro, Phys. Plasmas 6, 767 (1999).

[4] F. Mottez, Ann. Geophys. 22, 3033 (2004).

[5] C. Montagna and F. Pegoraro, Phys. Plasmas 14, 042103 (2007).

[6] M. G. Harrison and T. Neukirch, PRL 102, 135003 (2009).

[7] S. A. Lazerson, J. Plasma Phys. 77, 31 (2011).

[8] C. R. Stark and T. Neukirch, Phys. Plasmas 19, 012115 (2012).

[9] H. Tasso and G. N. Throumoulopoulos, J. Phys. A: Mathem. Theor. 40, F631 (2007).

[10] G. N. Throumoulopoulos, H. Tasso, H. Weitzner, Phys. Plasmas 13, 122501 (2006).

[11] P. Staeckel, Math. Ann. 35, 91 (1890).

[12] A. S. Eddington, Mon. Not. R. Astron. Soc. 76, 37 (1915).

[13] S. Chandrasekhar, Principles of Stellar Dynamics, reprinted by Dover, 1942.

[14] G. Contopoulos, Z. Astrophys. 49, 273 (1960).

[15] D. Lynden-Bell, Mon. Not. R. Astron. Soc. 124, 1 and 95 (1962).

[16] A. Hayli, Ann. Astrophys. 28, 49 (1965).

[17] G. Contopoulos, in Lectures in Applied Mathematics, Vol. 9 , p. 98 (1967).

[18] P. T. de Zeeuw, D. Lynden-Bell, Mon. Not. R. Astron. Soc. 215, 713 (1985). 
[19] P. T. de Zeeuw, Mon. Not. R. Astron. Soc. 215, 731; 216, 273; 216, 599 (1985).

[20] H. Dejonghe, Phys. Reports 133, 217 (1986).

[21] G. Bertin, M. Stiavelli, Reports on Progress in Physics 56493 (1993).

[22] G. Bertin, F. Pegoraro, F. Rubini, E. Vesperini, Astrophys. J. 434, 94 (1994).

[23] G. Bertin, Dynamics of Galaxies, Cambridge University Press (2000) (Chaps. 21-22).

[24] C. Efthymiopoulos, N. Voglis, and C. Kalapotharakos, Eur. Phys. J. Special Topics 186, 91 (2010).

[25] H. Tasso and G. N. Throumoulopoulos, Phys. Plasmas 18, 064507 (2011).

[26] F. Santini, H. Tasso, Internal Report IC/70/49, (1970). (See URL: streaming.ictp.trieste.it/preprints/P/70/049.pdf).

[27] D. Correa Restrepo and D. Pfirsch, Phys. Rev. E 55, 7449 (1997).

[28] D. Pfirsch, Z. Naturforsh. 39a, 1 (1984).

[29] P. J. Morrison and D. Pfirsch, Phys. Rev. A 32, 1714 (1985).

[30] D. Correa Restrepo and H. K. Wimmel, Physica Scripta 32, 552 (1985).

[31] D. Correa Restrepo, D. Pfirsch and H. K. Wimmel, Physica 136A, 453 (1986).

[32] R. G. Littlejohn, J. Plasma Phys. 29, 111 (1983).

[33] D. Pfirsch, P. J. Morrison, Phys. Fluids B 3, 271 (1991).

[34] G. N. Throumoulopoulos and D. Pfirsch, Phys. Rev. E 49, 3290 (1994).

[35] T. S. Hahm, Phys. Fluids 31, 2670 (1988).

[36] H. Sugama, Phys. Plasmas 7, 466 (2000).

[37] A. Brizard, Phys. Plasmas 7, 4816 (2000). 
[38] D. Pfirsch and D. Correa-Restrepo, J. Plasma Phys. 70, 719 (2004).

[39] D. Correa-Restrepo and D. Pfirsch, J. Plasma Phys. 70, 757 (2004).

[40] X. Lapillonne, B. F. McMillan, T. Görler, S. Brunner, T. Dannert, F. Jenko, F. Merz, and L. Villard, Phys. Plasmas 17, 112321 (2010).

[41] Feng Liu, Z. Lin, J. Q. Dong, and K. J. Zhao, Phys. Plasmas 17, 112318 (2010).

[42] H. S. Zhang, Z. Lin, H. Holod, X. Wang, Y. Xiao, and W. L. Zhang, Phys. Plasmas 17, 112505 (2010).

[43] Lu Wang and T. S. Hahm, Phys. Plasmas 17, 082304 (2010). 